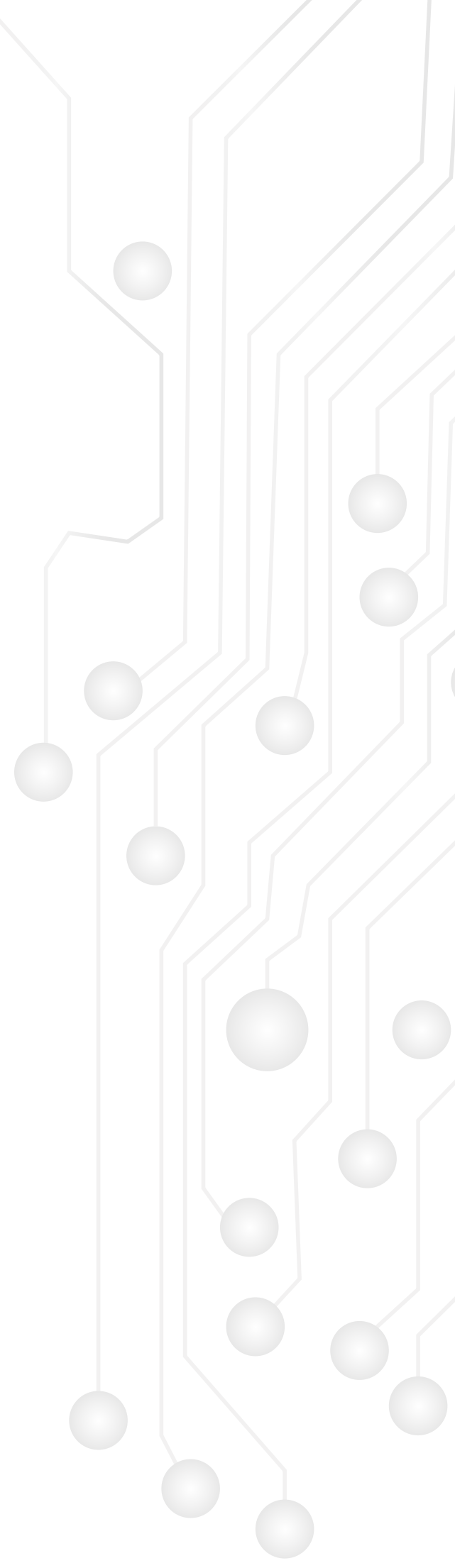




\section{PROPUESTA DE UN MODELO DE INTEGRACIÓN DE UNA RED INALÁMBRICA DE SENSORES EN UN AMBIENTE DE COMPUTACIÓN GRID}

PROPOSAL OF AN INTEGRATION MODEL OF A WIRELESS SENSOR NETWORK ON A GRID COMPUTING ENVIRONMENT

CARMEN INÉS BÁEZ-PÉREZ

Ingeniera de Sistemas

MSc. Ciencias de la Información y las Comunicaciones

Grupo de Investigación GISDYTEL

Universidad de Boyacá, Colombia

cibaez@uniboyaca.edu.co

FRANCISCO ARNALDO VARGAS-BERMÚDEZ

Ingeniero de Sistemas

MSc. Ciencias de la Información y las Comunicaciones

Grupo de Investigación GISDYTEL

Universidad de Boyacá, Colombia

franciscovargasb@uniboyaca.edu.co

MAURICIO OCHOA-ECHEVERRÍA

Ingeniero de Sistemas

Especialista en Telemática

Grupo de Investigación GISDYTEL

Universidad de Boyacá, Colombia m.ochoa@uniboyaca.edu.co

Recibido: 13/12/2013

Aceptado: 04/02/2014 


\section{RESUMEN}

Este artículo presenta los resultados de una propuesta de integración de una Red Inalámbrica de Sensores -RIS en un ambiente computacional GRID, el cual fue adaptado del modelo presentado por la Sensor Web Enablement (SWE). Una ventaja de esta integración es poder analizar los datos generados por la RIS, que por su cantidad deben ser tratados mediante el procesamiento distribuido que ofrece la GRID. Se plantea un modelo teórico que se describe por medio de la arquitectura general, presentado los principales componentes que hacen parte de su estructura. Este modelo servirá como base para una futura implementación de una aplicación que requiera los datos generados por una RIS.

Palabras Clave: Computación GRID, Redes Inalámbricas de Sensores, Servicios GRID, Sensor GRID

\section{ABSTRACT}

This article presents the results of a proposal of integration of wireless sensors (RIS) in a GRID computing environment, which was adapted from the model presented by the Sensor Web Enablement (SWE). An advantage of this integration is to be able to analyze the data generated by the RIS, that by their quantity should be treated by means of distributed processing offered by the GRID. There is a theoretical model which is described by means of the general architecture, presented the main components that are part of its structure. This model will serve as a basis for a future implementation of an application requiring a RIS-generated data.

Keywords: GRID computing, Wireless Sensor Networks, GRID Services, Sensor GRID. 


\section{INTRODUCCIÓN}

La computación GRID ofrece la posibilidad de compartir recursos heterogéneos tanto de hardware como de software. Por otra parte, las Redes Inalámbricas de Sensores - RIS generan grandes volúmenes de información que deben ser almacenados, para posteriormente extraerlos y procesarlos según las necesidades de los usuarios finales. Para optimizar el manejo que se le debe proporcionar a los datos se propone hacer uso de las bondades ofrecidas por la computación, es por ello que se plantea un modelo que permite integrar una RIS como un recurso informático dentro de una arquitectura GRID.

El modelo planteado permitirá la posibilidad de desarrollar prototipos que aseguren la convalidación del mismo y también diseñar componentes que permitan solventar problemas de manejo de datos generados por una RIS y necesarios para la toma de decisiones en diferentes áreas de la ciencia.

\section{METODOLOGÍA}

La metodología que se planteó para el desarrollo de la investigación, tuvo en cuenta los siguientes pasos:

\section{Etapa 1}

Revisión bibliográfica de los temas involucrados en el estudio:

- Redes inalámbricas de sensores

- Computación GRID

\section{Etapa 2}

De acuerdo con la información recopilada y analizada en la etapa 1 , se propuso un modelo que permita la incorporación de una red inalámbrica de sensores como un recurso de una ambiente de computación GRID. En esta etapa se describe la arquitectura del sistema mediante el uso de diagramas UML (Unified Modeling Lenguage - Lenguaje Unificado de Modelado), donde se definen cada uno de los diagramas que definen la estructura general del modelo y las funcionalidades que se proponen para una posterior implementación. 


\section{MODELO}

Una Red Inalámbrica de Sensores - RIS (Wireless Sensor Network - WSN), puede considerarse como miles, millones de dispositivos pequeños autónomos, distribuidos geográficamente, llamados nodos sensores, instalados alrededor de un fenómeno objeto para monitorizarlo, con capacidad de cómputo (realizar mediciones), almacenamiento y comunicación en una red conectada sin cable.

Uno de los componentes principales de una RIS es el SYNK, el cual es un nodo sumidero y/o Gateway, a donde todos los nodos sensores envían la información recolectada. Del SYNK posteriormente se pueden extraer los datos de la RIS mediante archivos planos o mediante conexiones directas con otras redes, donde se realizara el procesamiento y almacenamiento final de los datos. En una red típica de sensores, cada nodo del sensor funciona libremente y tiene un microprocesador y una cantidad pequeña de memoria para el proceso de señal y así programar una tarea específica. Cada nodo del sensor se comunica inalámbricamente con algunos otros nodos locales dentro de su gama de la radiocomunicación (Guibas, 2004).

La computación GRID tiene como uno de sus pilares el compartir la capacidad de cómputo, bases de datos y otras herramientas de forma segura (Globus consortium, n.a), buscando de esta manera aprovechar la disponibilidad de recursos heterogéneos de la capacidad instalada que se encuentra en las organizaciones, aprovechando este potencial para resolver problemas que demandan gran capacidad de procesamiento y almacenamiento (Allcock y Foster, sf.), (Wolfgang, s.f.), (Edinburgh, 2005-2008).

La GRID demanda un conjunto de elementos para su funcionamiento adecuado, tales como redes, nodos computacionales, software de infraestructura y estándares (Chicago, s.f.), también permite incluir nuevos elementos que generan datos.

El modelo que se propone plantea una propuesta que permite la integración de una RIS con un ambiente computacional GRID, con el objeto de adherir ésta como un recurso GRID, lo cual permite dar un tratamiento adecuado a los datos captados por dicha red y con ello ofrecer los mecanismos de recuperación y tratamiento de la información recolectada, esto permitirá optimizar los procesos que requieren de dicha información en diferentes áreas de aplicación.

La Figura 1, presenta el modelo general propuesto, el cual plantea a nivel general los diferentes componentes que se deberán tener en cuenta para la implementación de una arquitectura GRID que maneje como un recurso de generación de datos una RIS. El modelo plantea convertir en un recurso computacional la RIS, por medio de un Gateway, y generar un servicio GRID que permita el mapeo de los datos recolectados por la RIS a un mecanismo de persistencia (una base de datos o un archivo plano) que se encuentra instalado dentro de la GRID, de modo que a partir de éste se cuente con los datos para su extracción y tratamiento. 


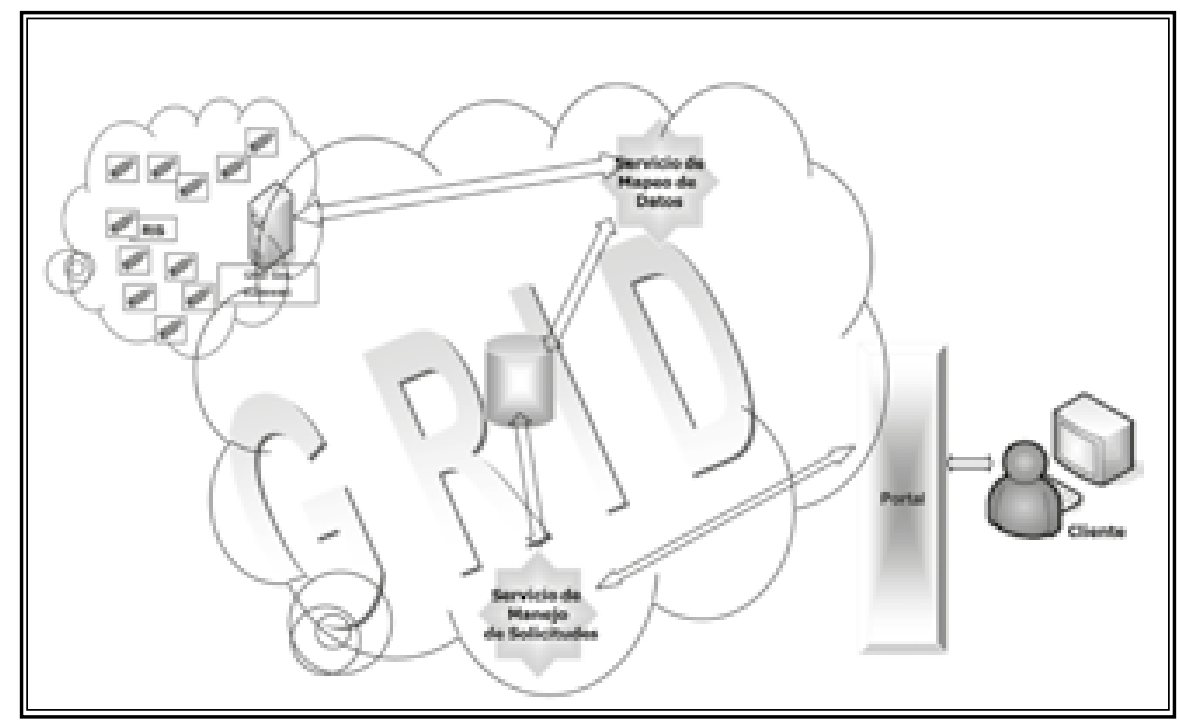

Figura 1. Modelo teórico para la integración de una RIS con un ambiente GRID. Fuente: (Open Geospatial Consortium Inc.. Adaptado por: GISDYTEL - Universidad de Boyacá., 2010)

Una vez los datos estén almacenados se podrán extraer y analizar por medio de un servicio de manejo de solicitudes, el cual será implementado dependiendo de las necesidades de información de los usuarios finales. Para que los usuarios finales puedan acceder a los datos procesados, se deberá implementar un portal web que garantice aspectos de seguridad de la información.

\section{ARQUITECTURA DEL MODELO PROPUESTO}

Con el fin de detallar el modelo teórico planteado, se hace una descripción general del mismo por medio de los diferentes elementos que componen su arquitectura. El problema que se busca solucionar con este modelo es la recolección de los datos generados por este tipo de redes, dentro de un ambiente de computación GRID, buscando que la RIS sea un recurso interno de la GRID y no solamente los datos que esta red genera, logrando así que toda la información que se maneje cumpla con las características de seguridad que se deben manejar en la red. Este artículo presenta una estructura general de la arquitectura propuesta en el modelo planteado.

La Figura 2 ilustra el modelo de proceso del negocio, con el fin de dar una visión general sobre los principales procesos que se deben tener en cuenta dentro de la implementación del mismo, allí se observan cada uno de los pasos que se deben realizar para ofrecer la posibilidad de recolección de información, iniciando con una petición del usuario, desarrollando una serie de tareas hasta lograr un resultado final. 


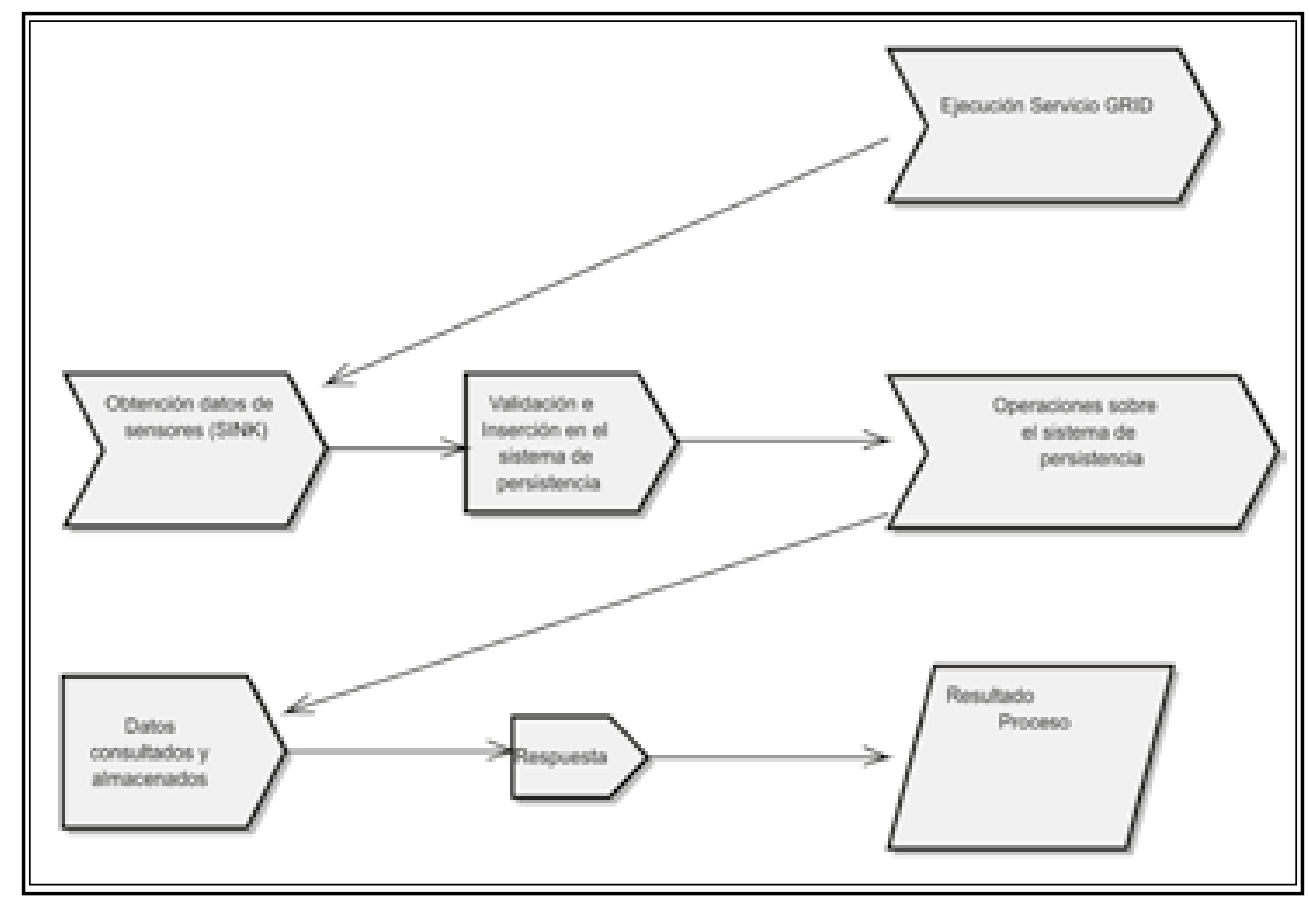

Figura 2. Modelo de proceso del negocio

Con el fin de realizar una mejor descripción del modelo general propuesto, se desarrollan los modelos de caso de uso; el primer modelo es el límite del sistema el cual se presenta en la Figura 3.

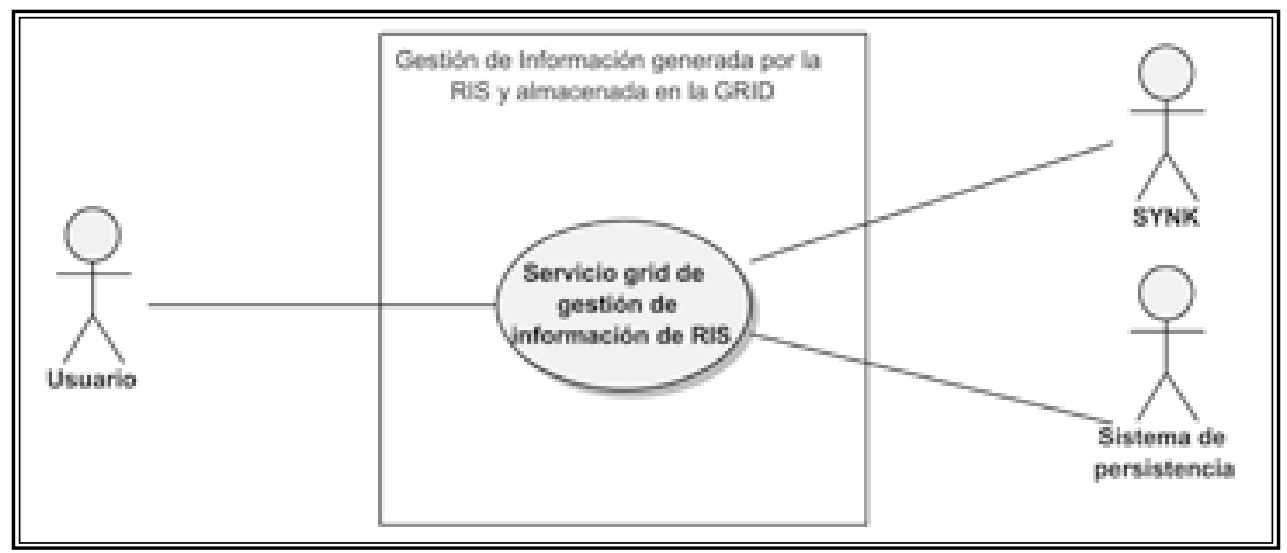

Figura 3. Límite del sistema propuesto 
El segundo caso de uso es el límite del sistema del servicio de integración, donde se desglosan los principales componentes del servicio de integración, este se presenta en la Figura 4.

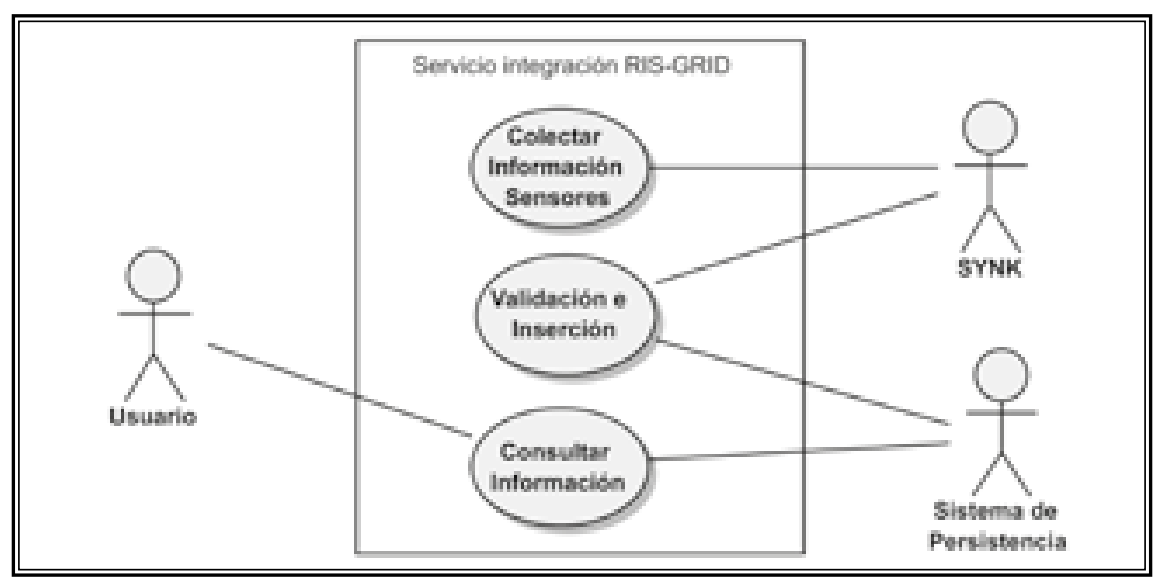

Figura 4. Límite del sistema del servicio de integración RIS-GRID

Una vez desglosado el límite del sistema, se detallan cada uno de los casos de uso que hacen parte del modelo, tal como se ilustra en la Figura 5.

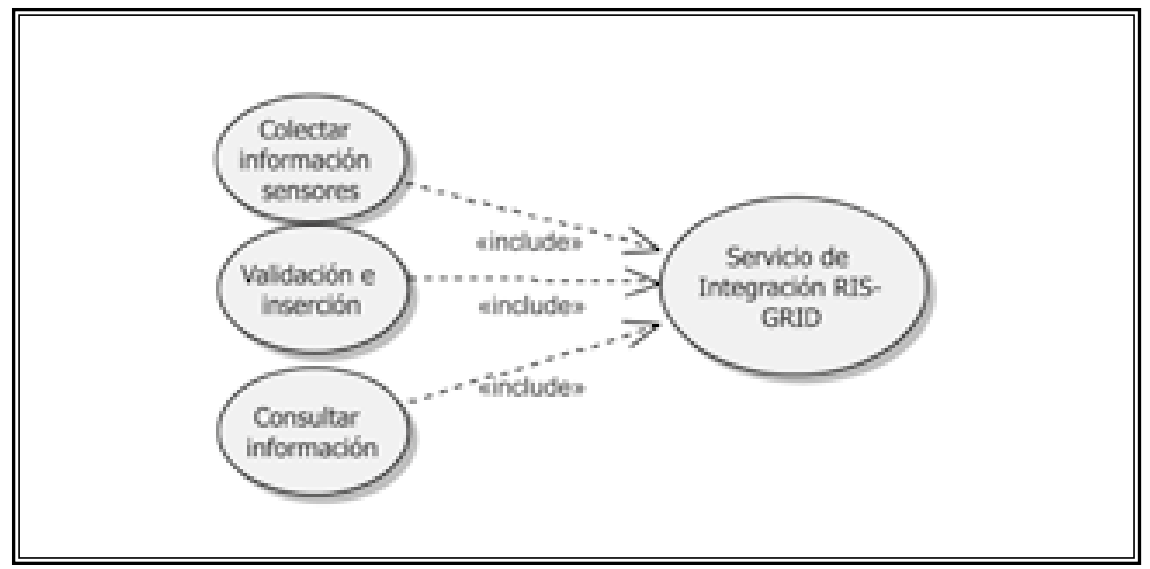

Figura 5. Diagrama de casos de uso general

El modelo propuesto plantea la recolección de información tomada desde el SYNK y llevada al sistema de persistencia seleccionado, previa validación de los datos recolectados. A continuación se detalla el caso de uso, en la Tabla 1. 


\begin{tabular}{|c|c|c|c|}
\hline CASO DE USO & \multicolumn{2}{|l|}{ General } & CU_1 \\
\hline PROPÓSITO & \multicolumn{3}{|c|}{$\begin{array}{l}\text { Permite la captura de los datos almacenados en el SYNK y la validación e inserción de } \\
\text { éstos en el sistema de persistencia. }\end{array}$} \\
\hline \multicolumn{4}{|c|}{ CURSO TÍPICO DE EVENTOS } \\
\hline \multicolumn{2}{|c|}{$\begin{array}{l}\text { Precondiciones } \\
\text { Datos almacenados en el SYNK. } \\
\text { Sistema de persistencia configurado. }\end{array}$} & \multicolumn{2}{|l|}{$\begin{array}{l}\text { Poscondiciones } \\
\text { Mensaje de proceso realizado correctamente }\end{array}$} \\
\hline \multicolumn{2}{|c|}{$\begin{array}{l}\text { Usuario } \\
\text { 1. El servicio de integración RIS-GRID se ejecuta periódi- } \\
\text { camente según parametrización establecida. }\end{array}$} & \multicolumn{2}{|c|}{$\begin{array}{l}\text { Solución } \\
\text { 2. El servicio consulta los datos almacenados en el SYNK. } \\
\text { 3. El servicio valida los datos. } \\
\text { 4. El servicio inserta los datos en el sistema de persistencia. } \\
\text { 5. El sistema de persistencia retorna un mensaje de ingreso } \\
\text { de los datos al servicio. }\end{array}$} \\
\hline
\end{tabular}

Tabla 1. Descripción del caso de uso general

Una vez se ha detallado el caso de uso general, se amplía la descripción de su funcionalidad mediante un diagrama de secuencia, el cual presenta el orden en que se ejecutan los procesos que realizan para que el servicio de integración cumpla con su objetivo. Aquí se presenta la forma en que se intercambian los mensajes entre los diferentes entes involucrados en la ejecución del servicio GRID. La Figura 6 permite observar dicho intercambio de mensajes, tanto de solicitud como respuesta. 


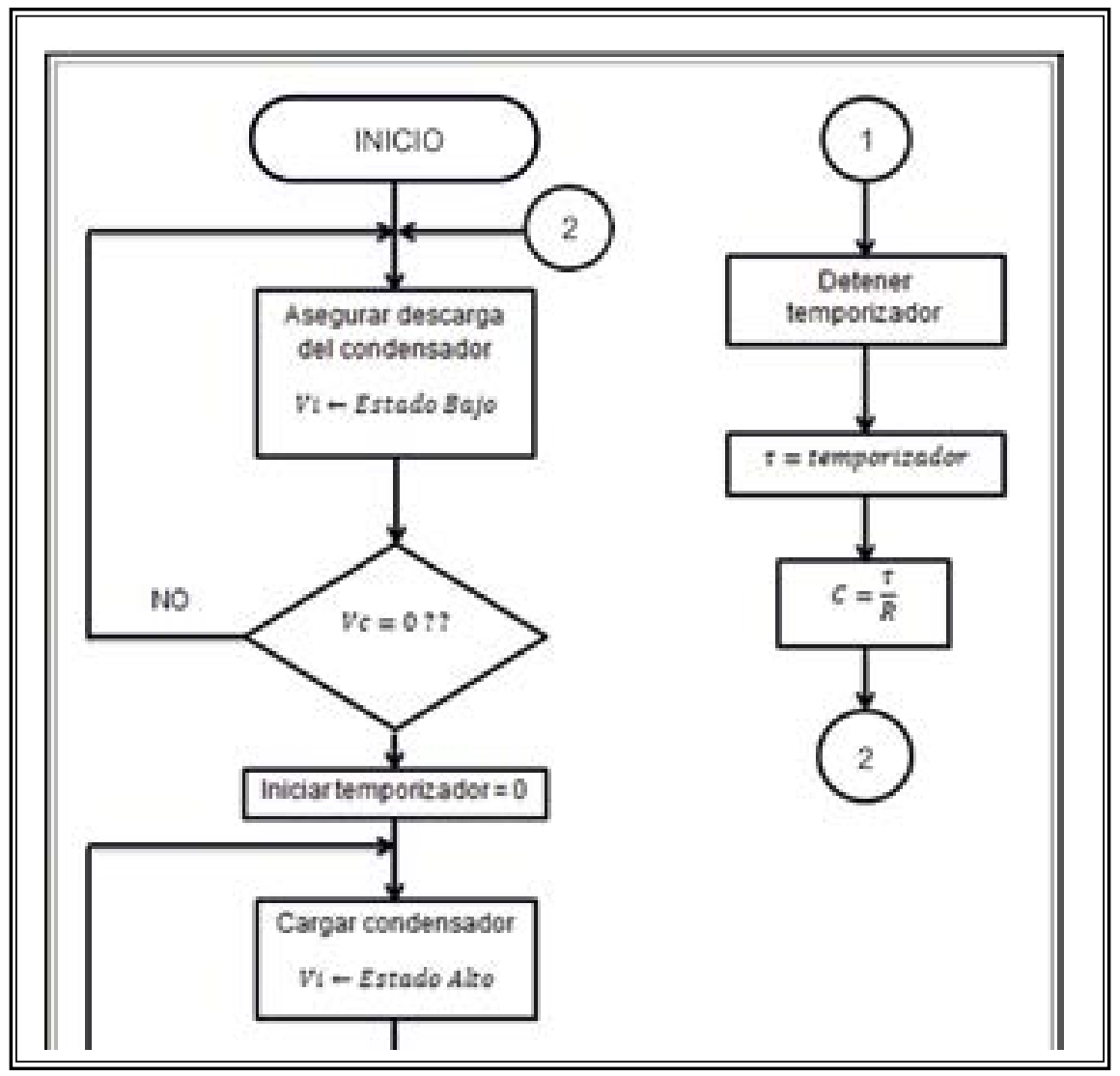

Figura 6. Diagrama de secuencia general

\section{Diagrama de Actividades}

Con el fin de ampliar el detalle del funcionamiento del caso de uso se define el diagrama de actividades que se muestra en la Figura 7, donde se presentan las diferentes tareas que se deben realizar para poder ejecutar las funcionalidades descritas. 


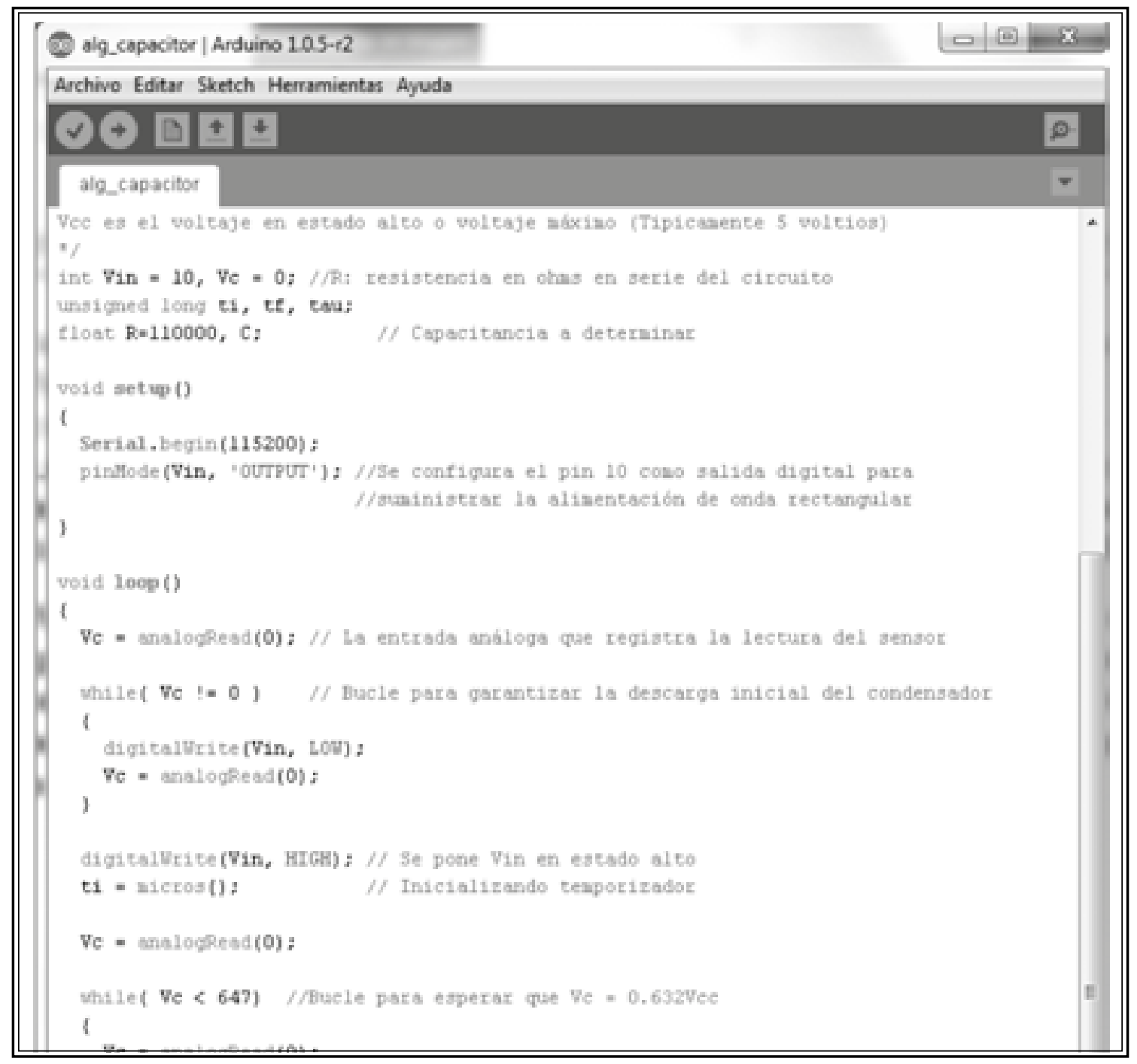

Figura 7. Diagrama de actividades

Una vez se describe el funcionamiento general del modelo propuesto, se procede a detallar cada uno de los casos de uso que conforman el sistema. Inicialmente se describe el caso de uso "Colectar información de sensores", el cual se detalla en Tabla 2. En seguida se describe el caso de uso "Validación e inserción", el cual se presenta en la Tabla 3. El caso de uso "Consultar información" se presenta posteriormente en Tabla 4. 


\begin{tabular}{|c|c|c|c|c|}
\hline CASO DE USO & \multicolumn{2}{|c|}{ Colectar información de sensores } & IDENTIFICADOR & CU_1.1 \\
\hline PROPÓSITO & \multicolumn{4}{|c|}{ Permite la captura de los datos almacenados en el SYNK. } \\
\hline \multicolumn{5}{|c|}{ CURSO TÍPICO DE EVENTOS } \\
\hline \multicolumn{2}{|c|}{$\begin{array}{l}\text { Precondiciones } \\
\text { Ubicación del SYNK } \\
\text { Datos almacenados en el SYNK. }\end{array}$} & \multicolumn{3}{|c|}{$\begin{array}{l}\text { Poscondiciones } \\
\text { Encontrar el SYNK } \\
\text { Datos Capturados }\end{array}$} \\
\hline \multicolumn{2}{|c|}{$\begin{array}{l}\text { Usuario } \\
\text { 1. El servicio de integración RIS-GRID se ejecuta periódi- } \\
\text { camente según parametrización establecida. }\end{array}$} & \multicolumn{3}{|c|}{$\begin{array}{l}\text { Solución } \\
\text { 2. El servicio busca la ubicación del SYNK. } \\
\text { 3. El servicio verifica que existan datos almacenados en } \\
\text { el SYNK. } \\
\text { 4. El servicio consulta los datos almacenados en el SYNK. }\end{array}$} \\
\hline
\end{tabular}

Tabla 2. Descripción del caso de uso colectar información de sensores

\begin{tabular}{|c|c|c|c|c|}
\hline CASO DE USO & \multicolumn{2}{|l|}{ Validación e inserción } & IDENTIFICADOR & CU_1.2 \\
\hline PROPÓSITO & \multicolumn{4}{|c|}{ Permite verificar la integridad de los datos leídos } \\
\hline \multicolumn{5}{|c|}{ CURSO TÍPICO DE EVENTOS } \\
\hline $\begin{array}{l}\text { Precondiciones } \\
\text { Datos capturados }\end{array}$ & & $\begin{array}{l}\text { Poscondiciones } \\
\text { Datos Validados }\end{array}$ & & \\
\hline \multicolumn{2}{|c|}{$\begin{array}{l}\text { Usuario } \\
\text { 1. El servicio de integración RIS-GRID valida la inte- } \\
\text { gridad de los datos y los inserta en el sistema de } \\
\text { persistencia. }\end{array}$} & \multicolumn{3}{|c|}{$\begin{array}{l}\text { Solución } \\
\text { 2. Lectura del archivo. } \\
\text { 3. Verifica que los datos estén en el formato ade- } \\
\text { cuado. } \\
\text { 4. Verifica que los datos estén completos. } \\
\text { 5. Datos incompletos son desechados. } \\
\text { 6. Datos completos pasan al siguiente proceso. } \\
\text { 7. Inserción de los datos en el sistema de persis- } \\
\text { 8. Envío. }\end{array}$} \\
\hline
\end{tabular}

Tabla 3. Descripción del caso de uso validación e inserción 


\begin{tabular}{|c|c|c|c|c|}
\hline CASO DE USO & \multicolumn{2}{|l|}{ Consultar información } & IDENTIFICADOR & CU_1.3 \\
\hline PROPÓSITO & \multicolumn{4}{|c|}{ Permite verificar la integridad de los datos leídos } \\
\hline \multicolumn{5}{|c|}{ CURSO TÍPICO DE EVENTOS } \\
\hline \multicolumn{2}{|c|}{\begin{tabular}{|l|} 
Precondiciones \\
Datos almacenados en el sistema de persistencia
\end{tabular}} & \multicolumn{3}{|c|}{$\begin{array}{l}\text { Poscondiciones } \\
\text { Resultados a las consultas realizadas }\end{array}$} \\
\hline \multicolumn{2}{|c|}{$\begin{array}{l}\text { Usuario } \\
\text { 1. El servicio de consulta recibe solicitudes y regresa } \\
\text { respuesta. }\end{array}$} & \multicolumn{3}{|c|}{ 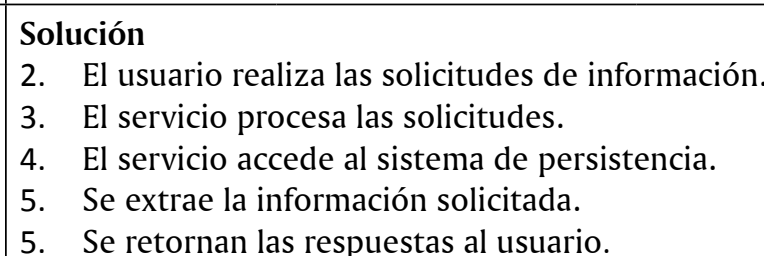 } \\
\hline
\end{tabular}

Tabla 4. Descripción del caso de uso consultar información

Una vez se han detallado cada uno de los casos de uso, se realiza el diagrama de secuencia para cada uno de ellos, con el fin de establecer la forma de interacción entre los diferentes elementos que participan en el desarrollo de los mismos. En la Figura 8 se presenta el diagrama de secuencia para el caso de uso general. En las Figuras 9, 10 y 11 se presentan los diagramas de secuencia para las funcionalidades principales. 


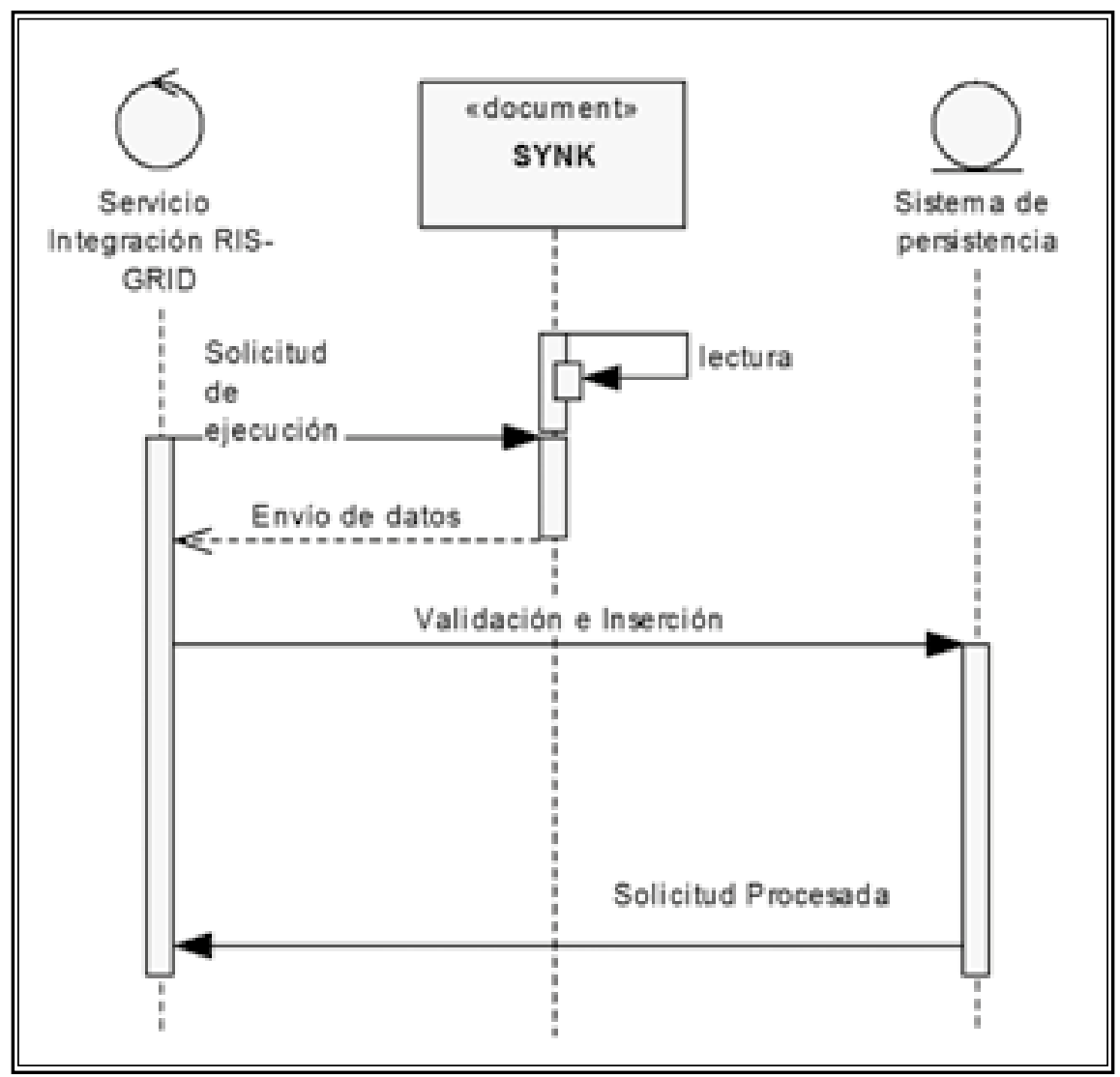

Figura 8. Diagrama de secuencia colectar información de sensores. 


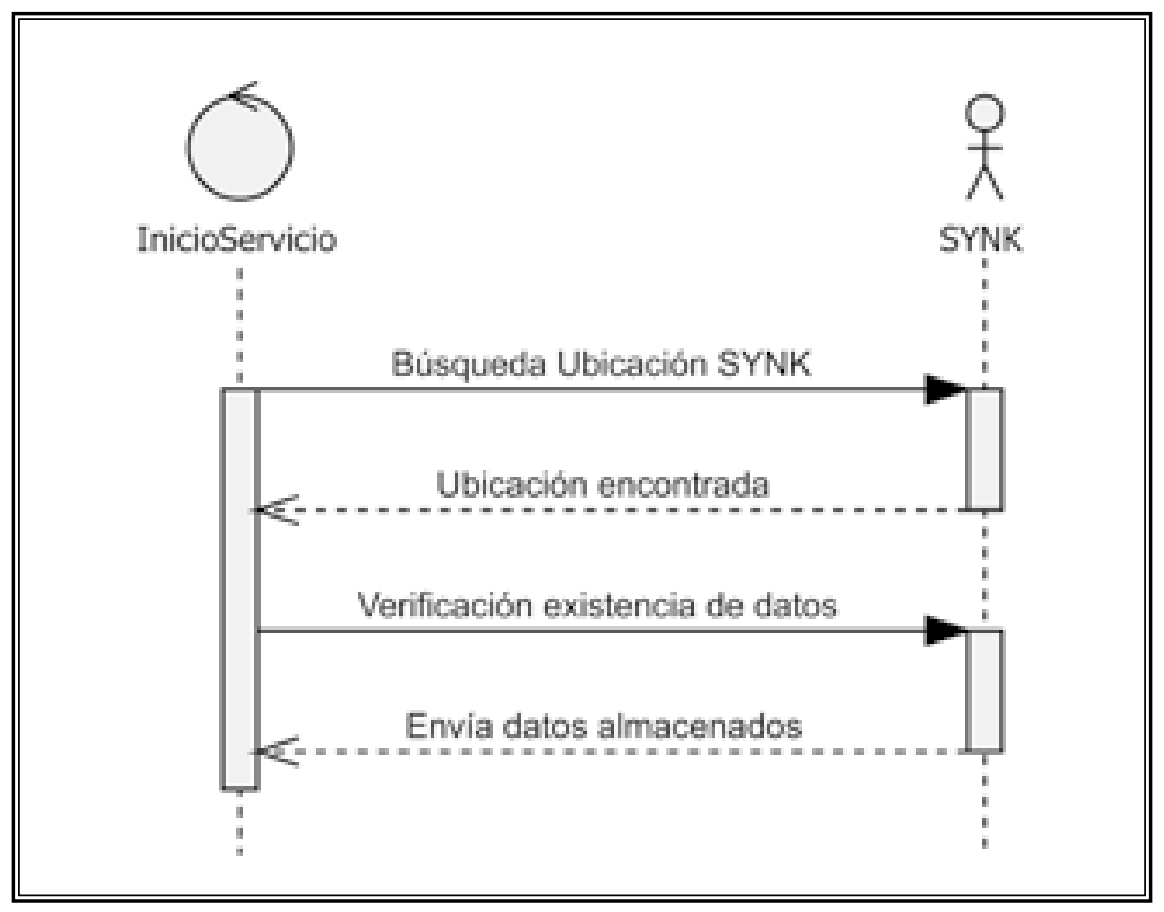

Figura 9. Diagrama de secuencia colectar información de sensores 


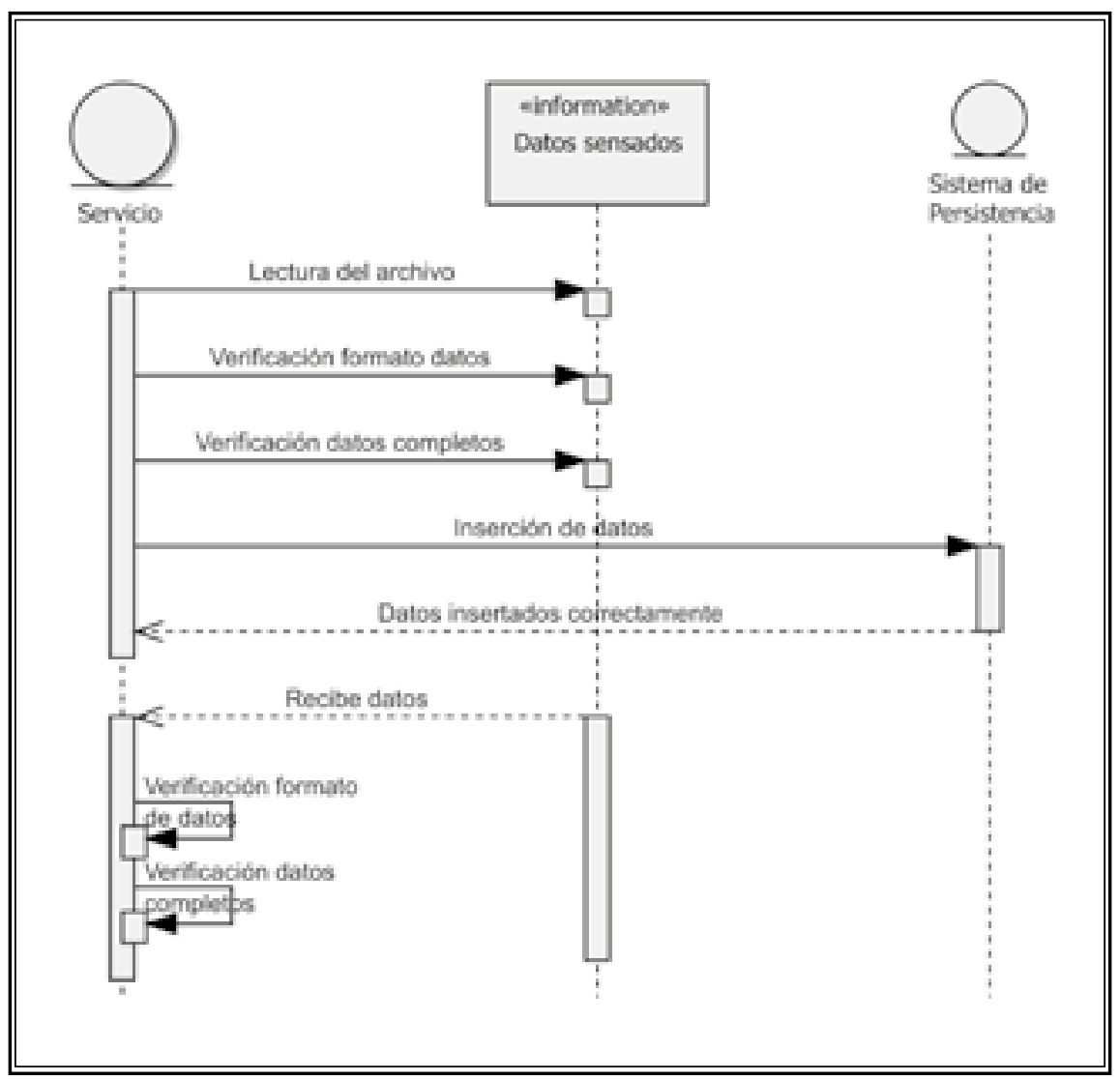

Figura 10. Diagrama de secuencia validación e inserción. 


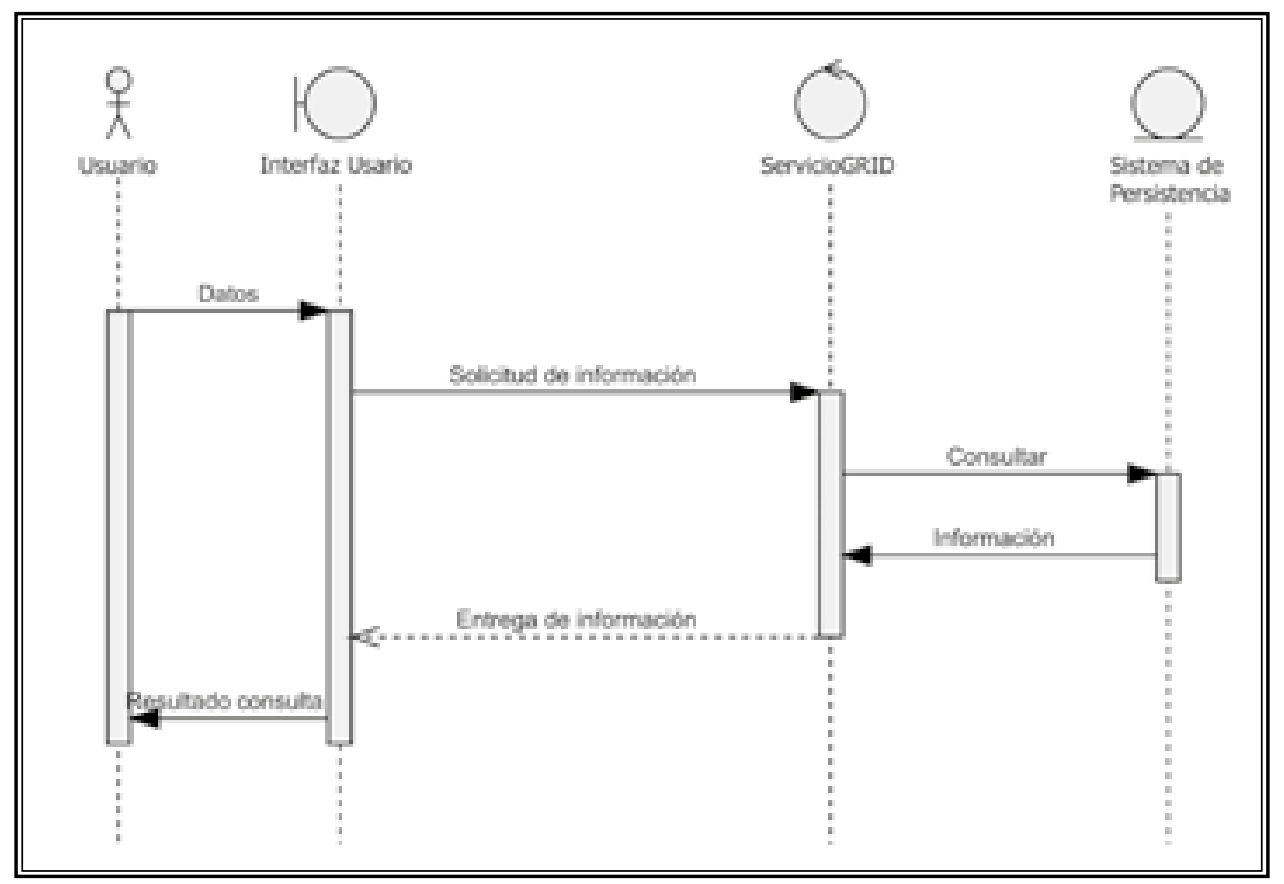

Figura 11. Diagrama de secuencia consulta de información.

Definidos los diagramas de secuencia para cada uno de los casos de uso, se diseñan los diagramas de actividades de los procesos principales definidos con el fin de ampliar la descripción de su funcionalidad. Estos diagramas se presentan en las Figuras 12, 13 y 14. 


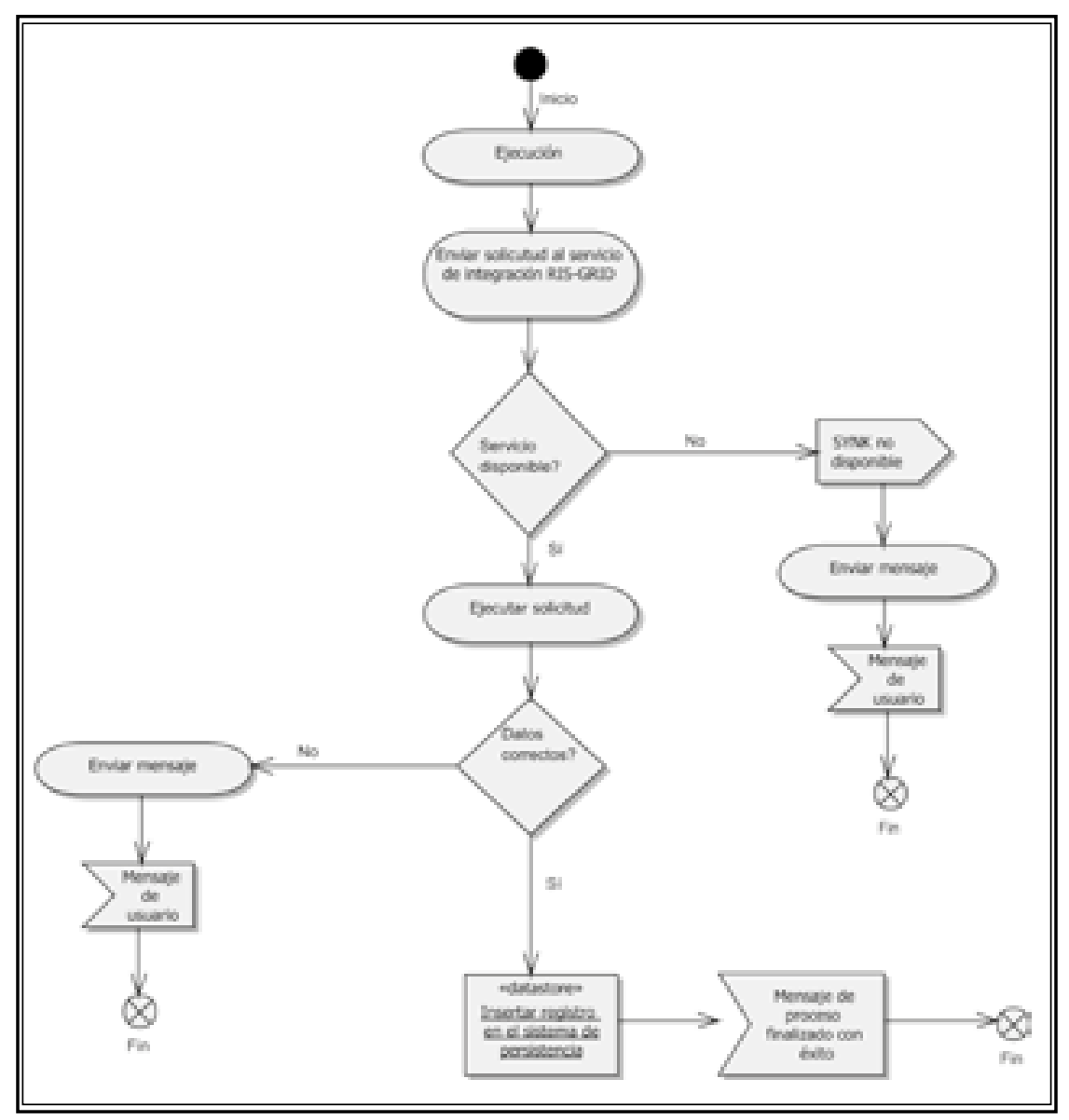

Figura 12. Diagrama de actividades colectar información de los sensores 


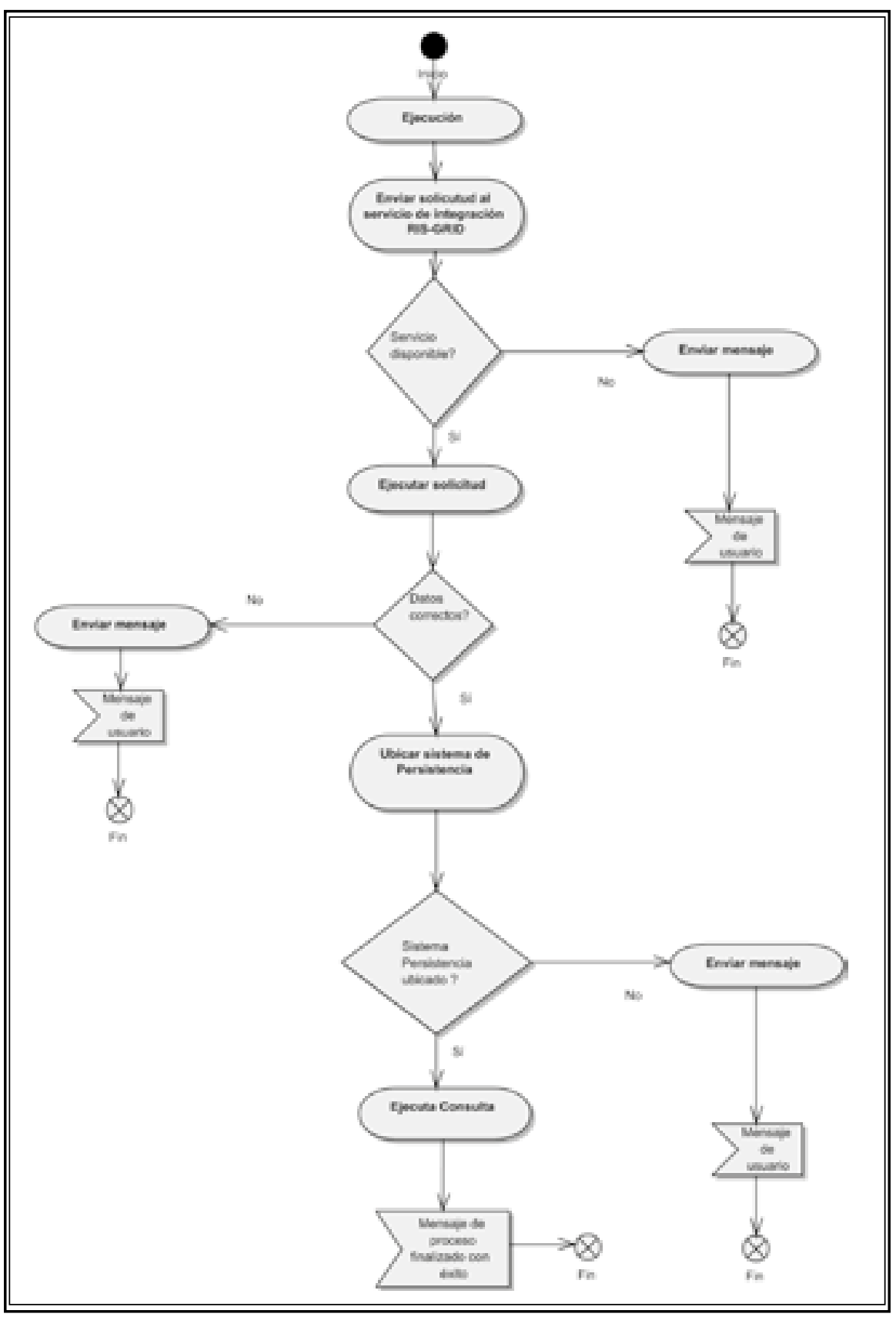

Figura 13. Diagrama de actividades validación e inserción 


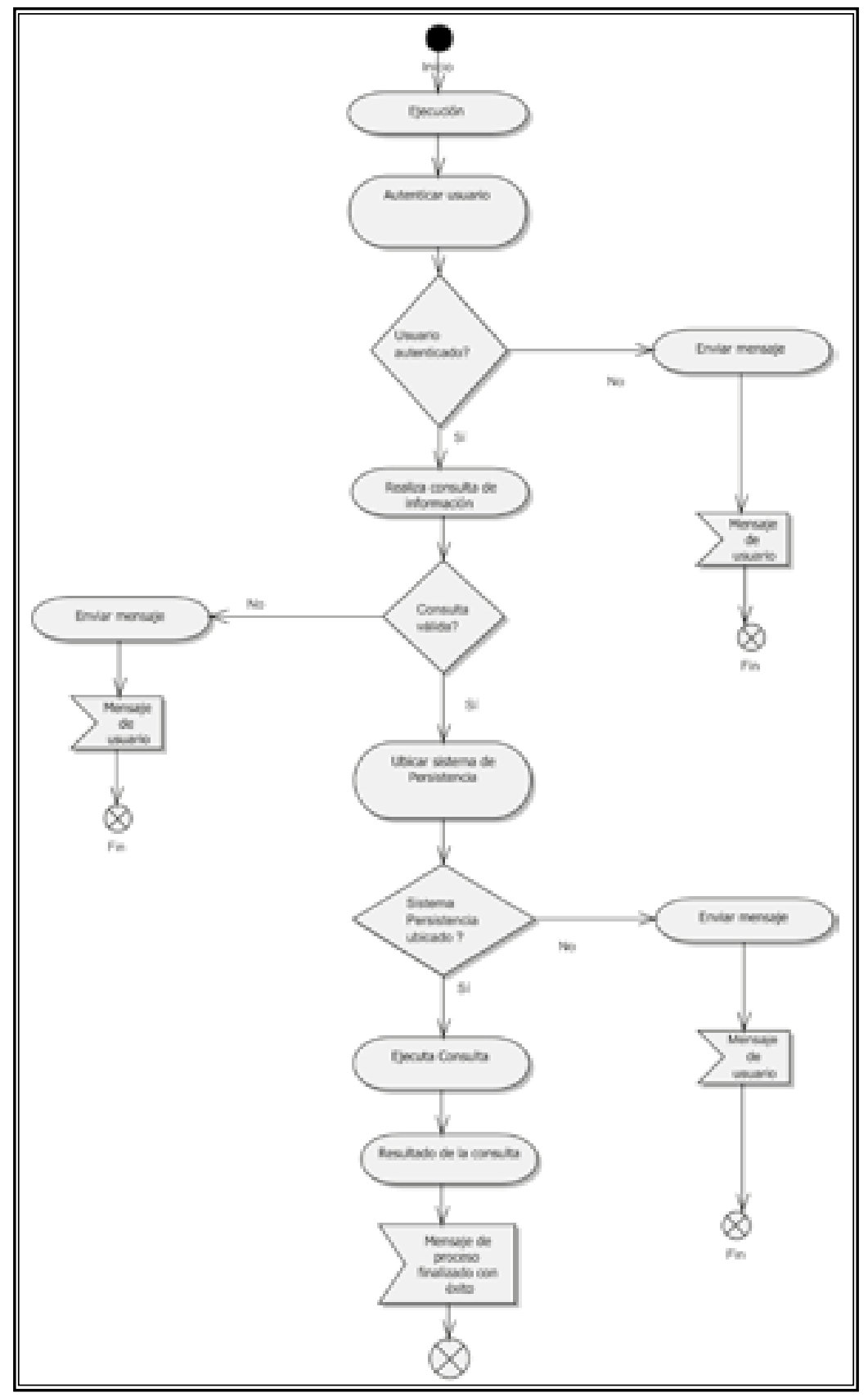

Figura 14. Diagrama de actividades consulta de información 


\section{Diagrama de clases}

Teniendo los diagramas anteriormente expuestos, se procede a realizar la abstracción del modelo que soportará las funcionalidades del proceso desde el punto de vista lógico y conceptual, tal como lo ilustra la Figura 15.

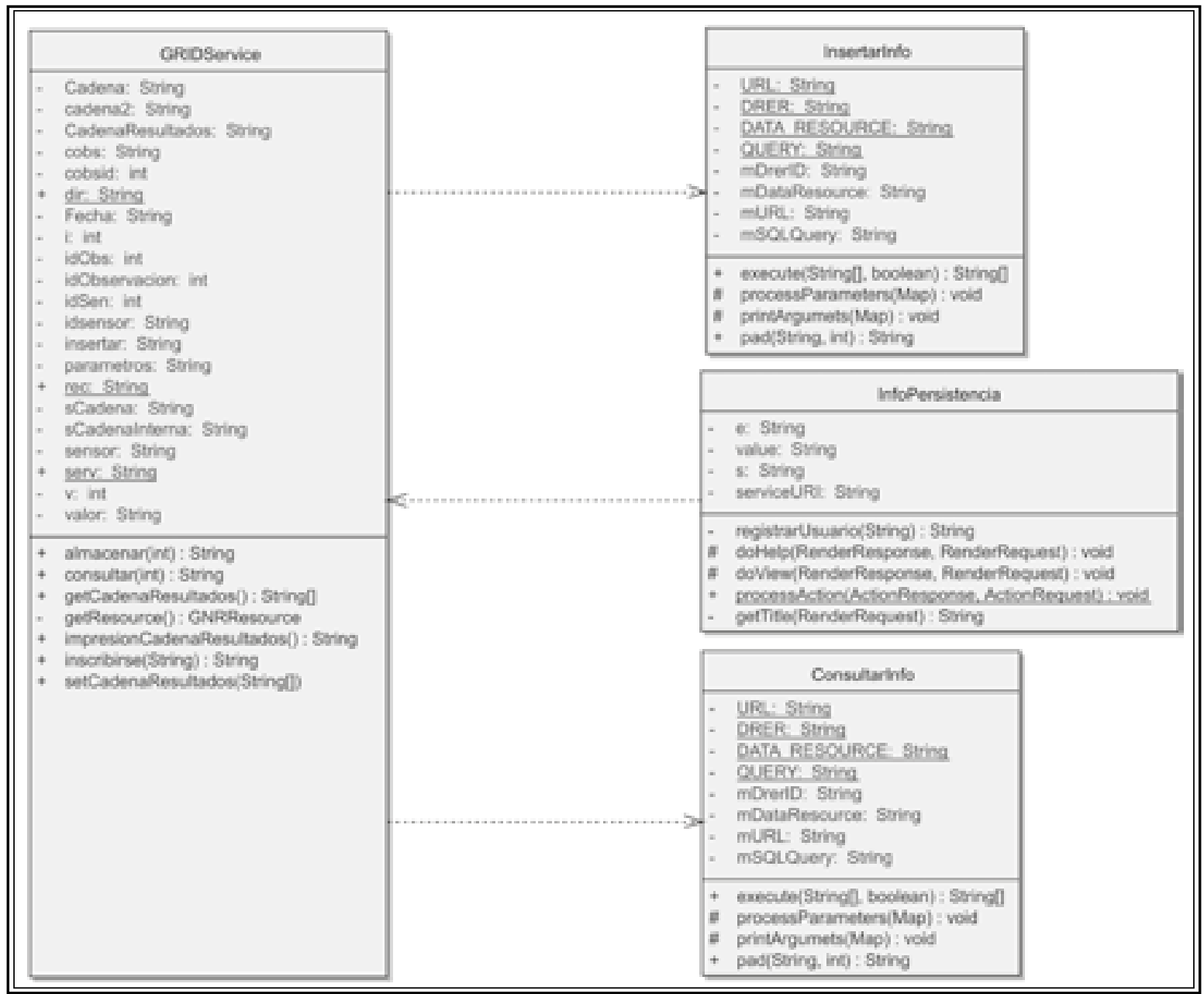

Figura 15. Diagrama de clases 


\section{Diagrama de componentes}

Las clases se organizan en componentes lógicos de acuerdo con las relaciones que estas tienen. Para el modelo propuesto se define una distribución de componentes que permitirá la interrelación entre los diferentes elementos involucrados, la que se presenta en la Figura 16.

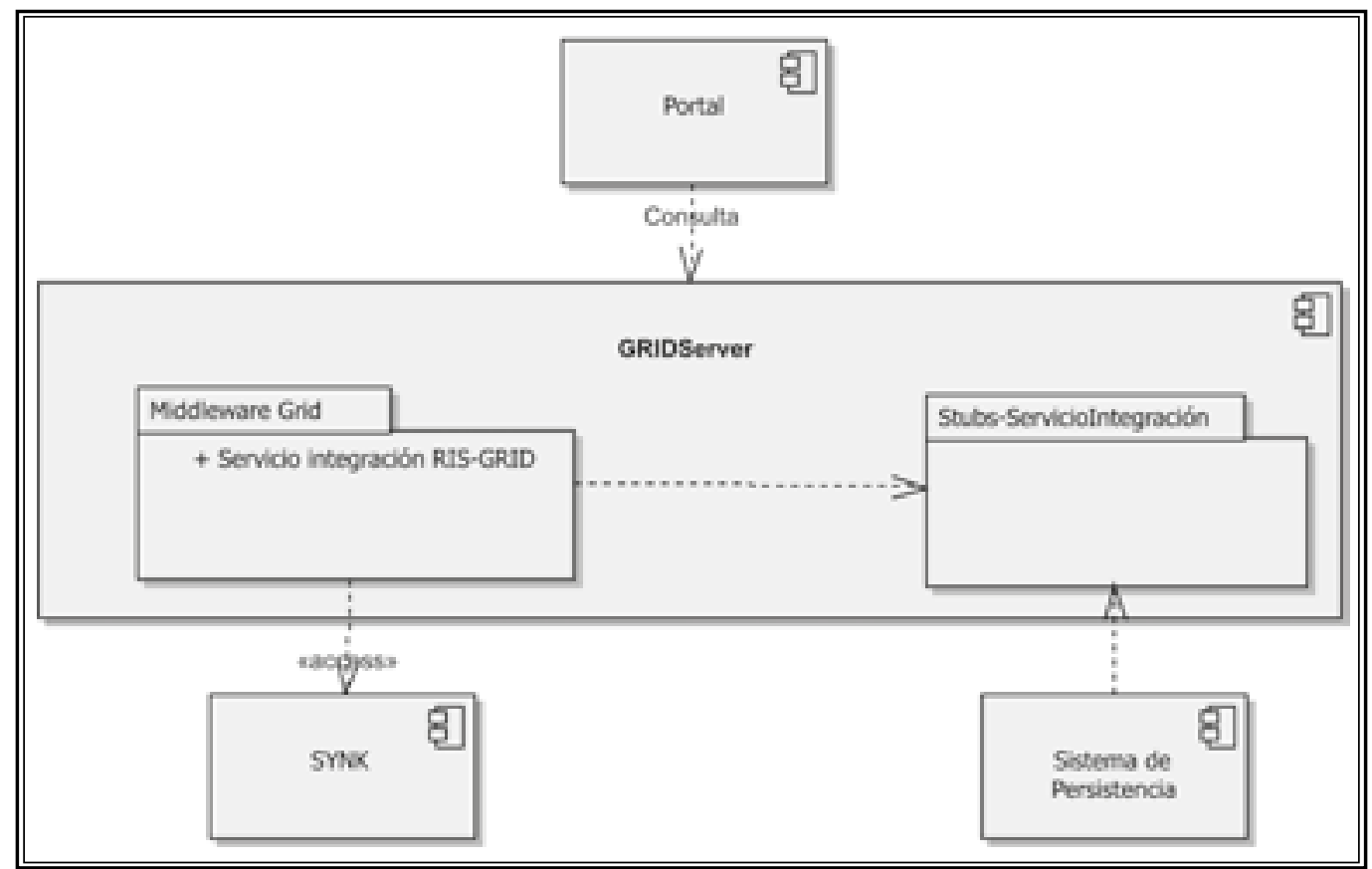

Figura 16. Diagrama de componentes

\section{Diagrama de paquetes}

Para una mejor organización, mantenimiento y escalabilidad se agrupan los componentes de acuerdo a su funcionalidad y la relación existente entre éstos, tal como se presenta en la Figura 17. 


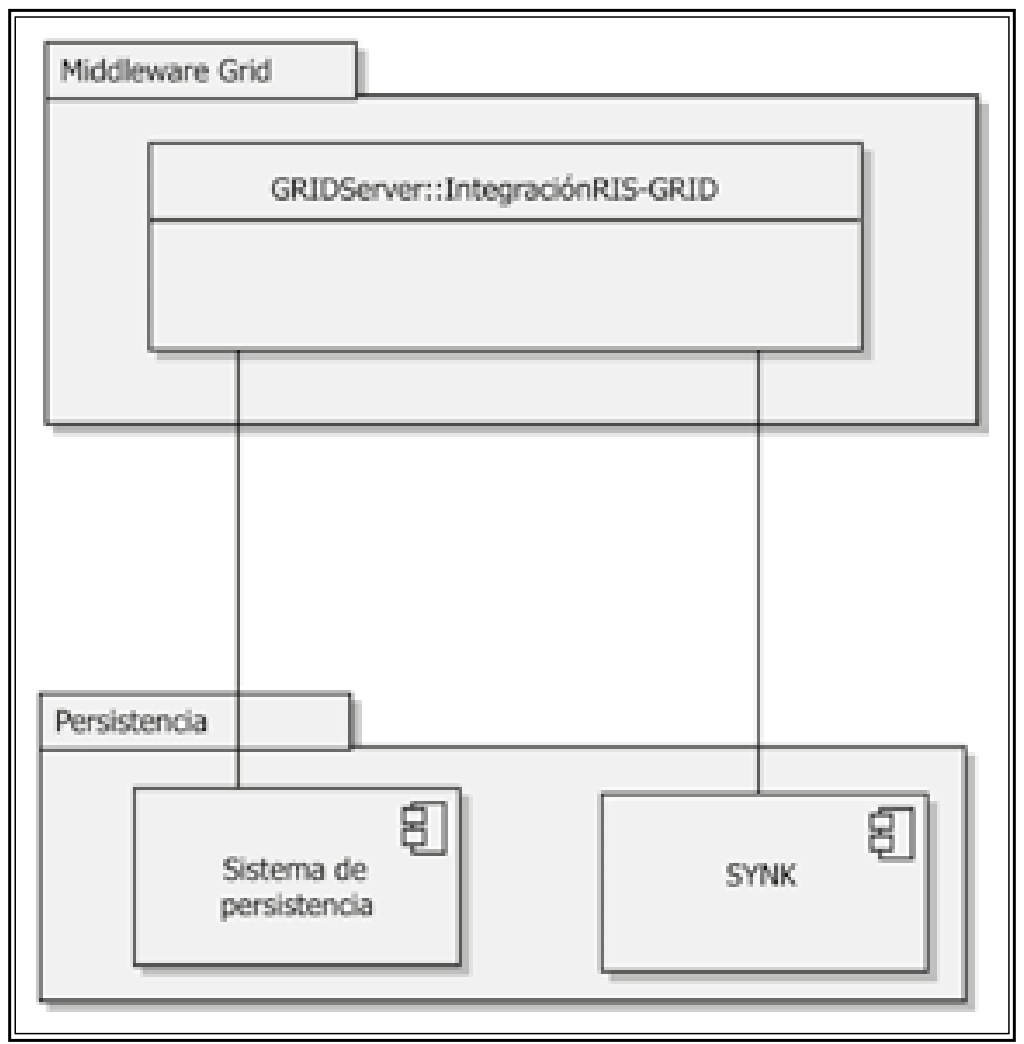

\section{Diagrama de despliegue}

Figura 17. Diagrama de paquetes

Define la arquitectura general de como deberá ser instalado el sistema teniendo en cuenta la infraestructura de Tecnologías de la Información que se requiere. La Figura 18 presenta un diagrama general de cómo se podría realizar dicha implementación. 


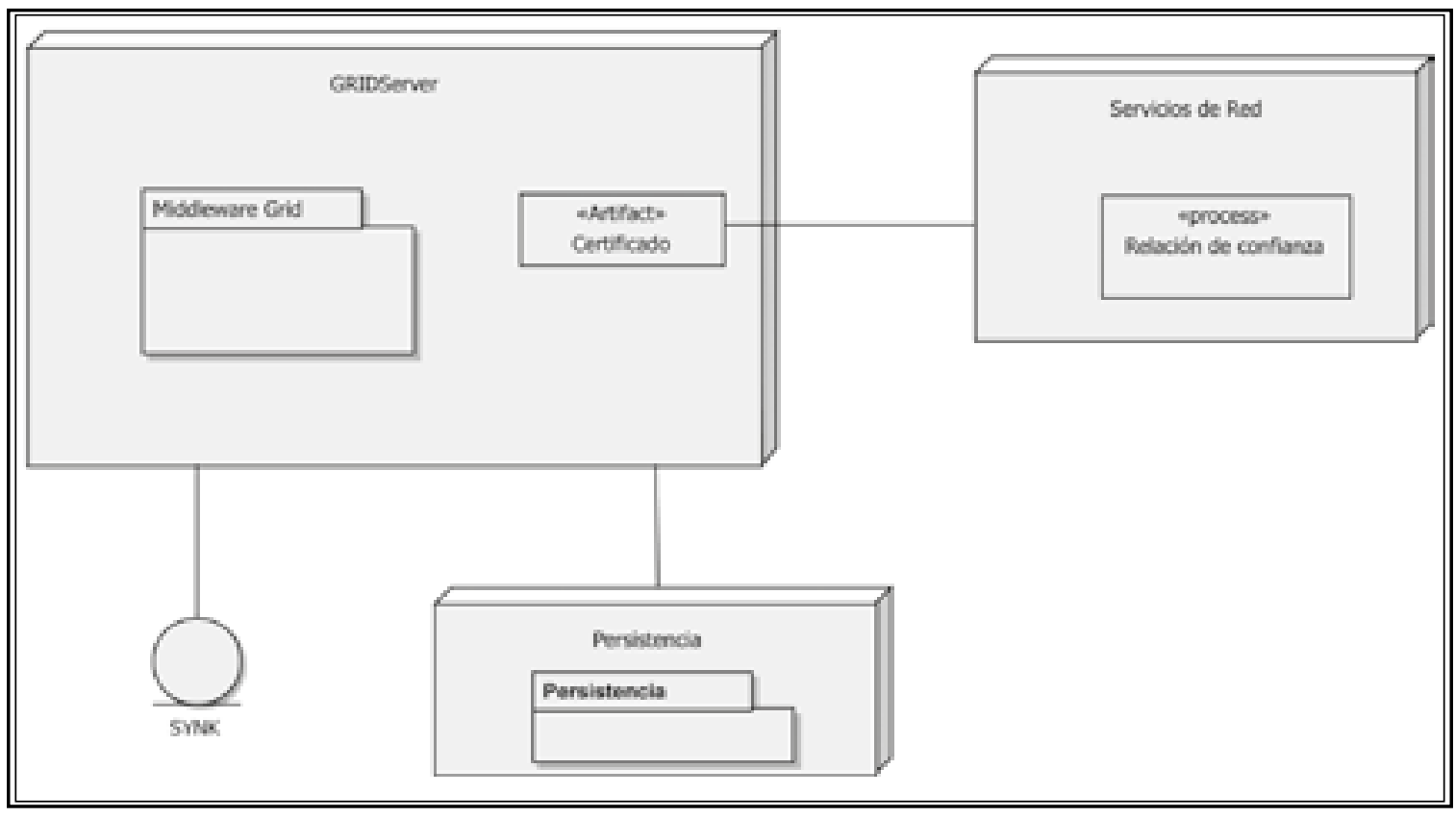

Figura 18. Diagrama de despliegue

\section{CONCLUSIONES}

La computación GRID ha surgido como una solución al manejo, almacenamiento y procesamiento de grandes volúmenes de datos, buscando de esta forma aprovechar los recursos que ya tienen disponibles los centros de investigación y centros académicos del mundo.

El modelo planteado, propone convertir como un recurso GRID una RIS, con el fin de mantener la integridad y seguridad de la red y poder dar un tratamiento adecuado a los datos generados por la RIS.

El modelo teórico permite un acercamiento a una futura implementación de un prototipo que permita convalidar dicho modelo y sirve de punto de partida para el desarrollo de trabajos en diferentes áreas que involucren el tratamiento de datos generados por una Red Inalámbrica de Sensores y que por su magnitud requieran procesamiento distribuido para su tratamiento. La arquitectura planteada permite proyectar las diferentes funcionalidades y alcance que se tendría al implementarla en un prototipo.

El modelo propuesto plantea el diseño de la interfaz entre dos tecnologías (redes inalámbricas de sensores y Computación), haciendo uso de dos servicios GRID, uno para el mapeado de los datos tomados 
desde el Gateway (SYNK) y transportados al mecanismo de persistencia y otro para permitir el manejo de las solicitudes de los datos que realicen los usuarios a través de un portal GRID.

Para dar una alternativa de solución a la gestión del gran volumen de datos generados en una red inalámbrica de sensores, el modelo propuesto incluye componentes, que permiten el almacenamiento, extracción y tratamiento de dichos datos con el objeto de generar información significativa para la toma de decisiones, de acuerdo a la naturaleza y necesidades de uso.

El modelo teórico planteado, permite un acercamiento a una futura implementación de un prototipo que permita convalidar dicho modelo y sirve de punto de partida para el desarrollo de trabajos en diferentes áreas que involucren el tratamiento de datos generados por una red inalámbrica de sensores.

La arquitectura planteada permite proyectar las diferentes funcionalidades y alcance que se tendría al implementarla en un prototipo. En ésta se detalla la forma como se obtienen los datos del sensor y las operaciones que se deben efectuar sobre el sistema de persistencia.

\section{Trabajos futuros}

Se propone realizar un prototipo que permita convalidar el modelo propuesto y de este modo poderlo proyectar en diferentes áreas de aplicación. En Boyacá se pueden solucionar múltiples problemas de manejo masivo de datos por medio de la implementación de sistemas GRID, lo cual redundaría en mejor gestión y aprovechamiento de información, en pro de la toma oportuna de decisiones que llevarían mejoramiento de los procesos ambientales que se dan en nuestro ecosistema.

Aprovechar la infraestructura tecnológica en cuento a hardware, redes de comunicaciones y software que actualmente ofrece la Corporación Red de Instituciones de Educación, Investigación y Desarrollo del Oriente Colombiano -UNIRED- con el fin de plantear proyectos de investigación conjuntos, compartiendo recursos computacionales, académicos, bibliográficos, entre otros.

Se propone realizar trabajos de investigación relacionados con el estudio de técnicas de recolección de datos y extracción de información, que permitan optimizar el tratamiento de los mismos y la entrega de información a usuarios finales. Se propone como proyecto adicional la elaboración de un estudio de factibilidad técnico-económica que permita determinar la viabilidad del montaje de un laboratorio GRID en la Universidad de Boyacá. 


\section{REFERENCIAS BIBLIOGRÁFICAS}

Allcock, W. E., y Foster, I. y. (s.f.). Reliable data transport : a critical service for the GRID. (U. o. Chicago, Ed.) Recuperado el 20 de 05 de 2011, de http://www.globus.org/alliance/publications/papers/ GGF11_RFTV-Final.pdf

Chicago, U. o. (s.f.). The Globus Alliance. Welcome to Globus. Recuperado el 12 de 06 de 2011, de http://www.globus.org

Edinburgh, T. U. (2005-2008). OGSADAI. The Ogsadai Project. Recuperado el 14 de 08 de 2012, de http://www.ogsadai.org/

Guibas, F. Z. (2004). Wíreles Sensor Networks: An Information Processing Approach. IEEE Magazine, 13-17.

Globus Consortium. (n.a). http://www.globus.org/toolkit/about.html. Recuperado el 2012 de 10 de 22, de About Globus Toolkit: http://www.globus.org/toolkit/about.html

Open Geospatial Consortium INC. Adaptado por: GISDYTEL - Universidad de Boyacá. (2010). OpenGIS ${ }^{\circledR}$ Sensor Web Enablement Architecture Document. Mike Botts, Alex Robi, John Davidson y Ingo Simonis.

Wolfgang, H. (s.f.). Data management in an international data GRID project. 